\title{
Time ephemeris
}

\author{
TOSHIO FUKUSHIMA \\ National Astronomical Observatory \\ 2-21-1, Ohsawa, Mitaka, Tokyo 181, JAPAN \\ (Internet) toshio@spacetime.mtk.nao.ac.jp
}

(To be appeared in Astron. Astrophys.)

The location-independent part of TCB-TCG, the difference between the two new time scales adopted by the IAU (1992), was integrated numerically for three JPL planetary/lunar ephemerides; DE102, DE200, and DE245. The differences among these three integrations were mostly explained by the difference in the adopted constants of the ephemerides. It was shown that the post-Newtonian correction and the perturbation by asteroids are negligible except for the mean rate. $L_{C}$. The comparison of these numerical integrations with the analytical formulas of Hirayama et al. (1987) and Fairhead and Bretagnon (1990) as well as their extended versions lead to the best estimate of $L_{C}$ as

$$
L_{C}=L_{C}^{(\mathrm{TE} 245)}+\Delta L_{C}^{(\mathrm{PN})}+\Delta L_{C}^{(\mathrm{A})}=1.4808268457 \times 10^{-8} \pm 1.0 \times 10^{-17} .
$$

Combining this with the recent value of the geoid potential in Bursa et al. (1992), we estimated the value of $L_{B}$, the scale difference between TCB and TT, as

$$
L_{B}=1.550519748 \times 10^{-8} \pm 4 \times 10^{-17} .
$$

Table I summarizes these conclusions. These estimates of $L_{C}$ and $L_{B}$ are more reliable than the former values we gave (Fukushima et al. 1986). The new estimate of $L_{B}$ will be useful in converting the numerical values of some precisely determined astronomical constants such as AU measured in meter from those in TDB to those in TCB. Also the numerically integrated TCB-TCG, which are to be called Time Ephemeris, will be useful when converting between TCB and TDB, i.e. the time scales themselves.

\section{TABLE I}

Estimate of $L_{C}$ and $L_{B}$

\begin{tabular}{llrrl}
\hline Item & Meaning & Value & $\sigma$ & Note \\
\hline & Main part & 1480826856.21 & 0.50 & TE245 \\
& Post-Newtonian correction & -10.97 & 0.01 & TE245PN \\
& Asteroid perturbation & +0.45 & 0.5 & TE245A \\
\cline { 2 - 5 }$L_{C}$ & Mean rate of TCB-TCG & 1480826845.7 & 1.0 & Sub Total \\
$L_{G}$ & Mean rate of TCG-TT & 69692901.9 & 3 & Bursa et al. (1992) \\
\cline { 2 - 5 }$L_{B}$ & Mean rate of TCB-TT & 1550519748 & 4 & Total \\
\hline
\end{tabular}

Notes: The unit is $10^{-17}$. 
Fig. 2. Periodic part of TE200-TE245

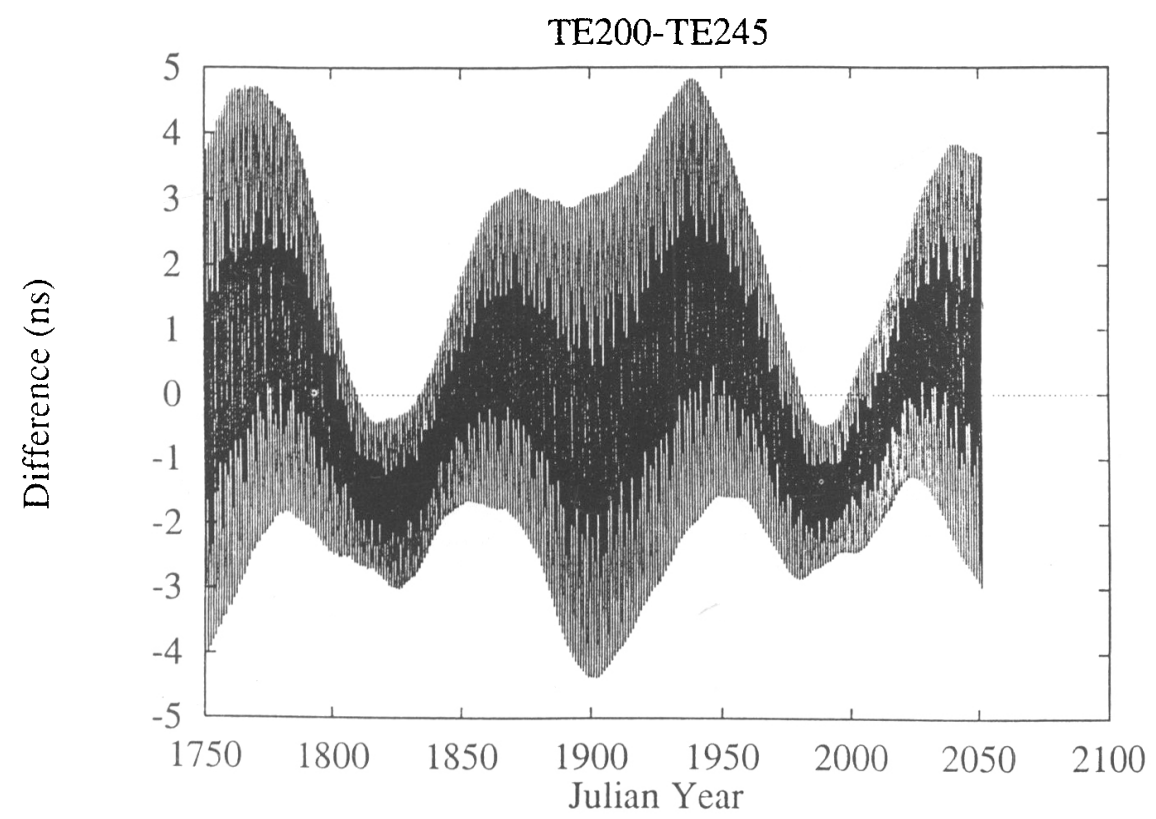

Fig. 10. Periodic part of TE245-FB2R for 1980-2000

TE245-FB2R

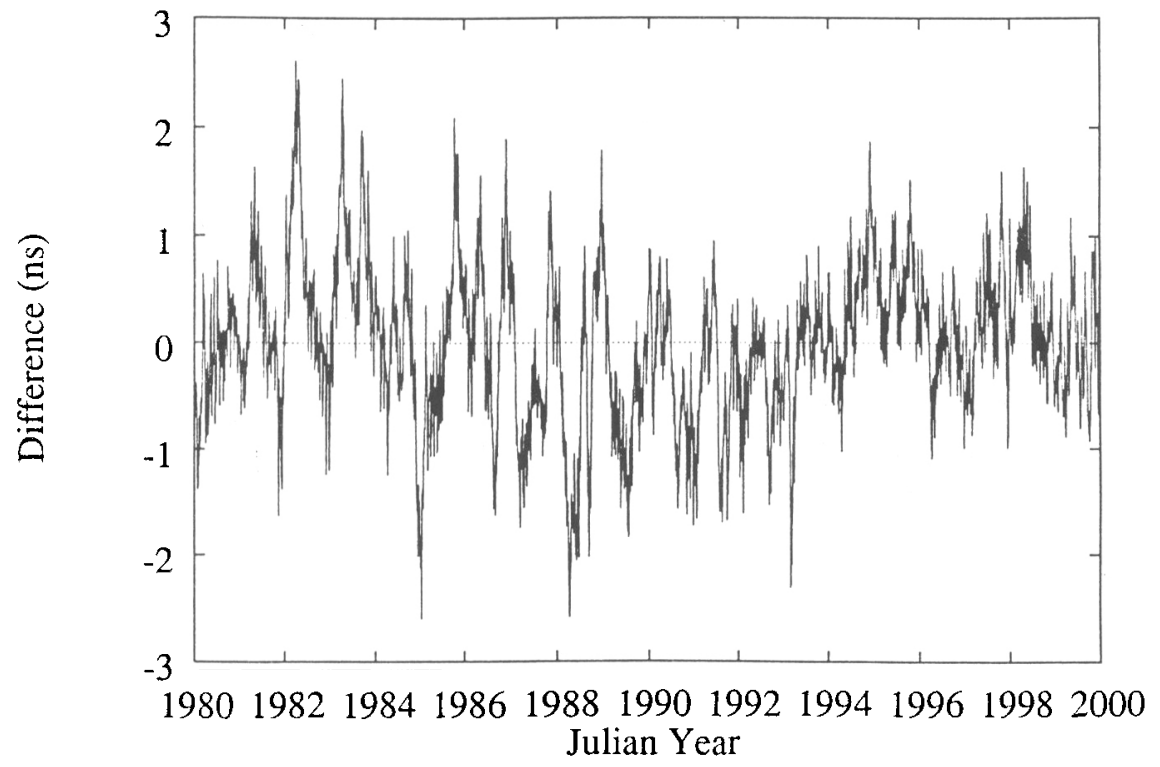

